\title{
Post partum haemorrhage and the WOMAN Trial
}

Post partum haemorrhage (PPH) is a leading cause of maternal mortality, accounting for about 100,000 maternal deaths every year. ${ }^{1}$ Almost all $(99 \%)$ of the deaths are in low and middle income countries and most of the deaths occur in the immediate post-partum period. ${ }^{2}$ There are known effective treatments for preventing severe bleeding after childbirth and it is essential to ensure that all pregnant women have access to these. ${ }^{3}$ However, even with these treatments, many women will develop severe post partum bleeding and there is an urgent need for a safe and effective treatment that is inexpensive and easy to administer across a range of different healthcare settings. This editorial invites the participation of obstetricians, midwives and nurses from around the world to join an international collaborative effort to identify such a treatment.

Tranexamic acid (TXA) is an anti-fibrinolytic agent that inhibits clot breakdown by blocking the lysine binding sites on plasminogen molecules. ${ }^{4}$ TXA is not a new drug and has been used for many years to treat menorrhagia and dental extraction in people with haemophilia. More recently, it has been used to reduce bleeding in elective surgical patients. Although there are many on-going trials of TXA in elective surgery, the accumulated evidence already shows that TXA reduces bleeding. A recently updated systematic review of randomised trials of TXA in elective surgery found 53 randomised trials, including a total of 3,836 participants. TXA reduces blood transfusion by about one third $(R R=0.61$, $95 \% \mathrm{Cl} 0.54$ to 0.70 ) with a trend towards reduced mortality that was not statistically significant $(\mathrm{RR}=0.6195 \% \mathrm{Cl} 0.32 \text { to } 1.12)^{5}$

New results from the CRASH-2 trial of TXA in bleeding trauma patients provide exciting grounds for optimism that TXA might reduce mortality and the need for hysterectomy in women with $\mathrm{PPH}{ }^{6,7}$ In the CRASH-2 trial, 20,211 adults with traumatic haemorrhage were randomised to either TXA (loading dose 1 gram over ten minutes followed by infusion of 1 gram over 8 hours) or matching placebo, with $99.6 \%$ follow-up. The risk of death due to bleeding was significantly reduced with TXA $(489 / 10,060$ [4.9\%] TXA versus $574 / 10,067$ [5.7\%] placebo; relative risk 0.85 [95\% $\mathrm{Cl} 0.76$ to 0.96 ]; $2 \mathrm{P}=0.008$ ). Importantly, there was no increase in fatal or non-fatal vascular occlusive events (168 [1.7\%] TXA versus 201 [2.0\%] placebo; relative risk 0.84 [95\% $\mathrm{Cl} 0.68$ to 1.0 ]; $2 \mathrm{P}=0.08$ ). All cause mortality was also significantly reduced with TXA $(1,463$ [14.5\%] TXA versus 1,613 [16.0\%] placebo; $R R=0.91$ [95\% Cl 0.85 to 0.97 ]; $2 \mathrm{P}=0.004$ ). The large numbers of patients studied from hospitals in high, middle and low income countries, help these results to be generalised widely. Because TXA is inexpensive and easy to administer, it can easily be added to the normal medical and surgical management of trauma patients in hospitals world-wide.

The knowledge that TXA safely reduces mortality in traumatic haemorrhage raises the possibility that it might also be effective in PPH. To date, there have been only a few small trials of TXA in post partum bleeding, including in total less than 500 women. ${ }^{8}$ Although there was a significant reduction in post partum blood loss in women treated with TXA, the quality of the trials was poor. None had well concealed treatment allocation and even in aggregate they were too small to assess the effects of TXA on mortality, hysterectomy and thrombotic side effects. The recently updated PPH treatment guidelines prepared by the World Health Organization (WHO) state that TXA may be used if other measures fail, but points out that the evidence base is poor and calls for further clinical trials of TXA in $\mathrm{PPH}$.

The WOMAN (World Maternal Antifibrinolytic) trial, coordinated by the London School of Hygiene and Tropical Medicine (University of London), has been launched in response to this important clinical uncertainty. WOMAN is a pragmatic, randomised, double blind, placebo controlled trial among women with a clinical diagnosis of postpartum haemorrhage that will determine reliably the effect of the early administration of TXA on death, hysterectomy and other morbidities (surgical interventions, blood transfusion, risk of non-fatal vascular events), in woman with PPH. The trial is already recruiting eligible women in hospitals in Africa and Europe but many new collaborating centres are needed in 
order to reach the target sample size of 15,000 women.

Full details of the protocol and how to join the trial are available on the trial website (), or by e-mailing TheWomanTrial@Lshtm.ac.uk. The success of the WOMAN Trial depends on the collaboration of midwives, nurses and doctors from hospitals all around the world. The hope is that by working together we might identify a safe and effective treatment that could save the lives of tens of thousands of new mothers.

\section{REFERENCES}

1. Hogan MC, Foreman KJ, Naghavi M, et al. Maternal mortality for 181 countries, 1980-2008: a systematic analysis of progress towards Millennium Development Goal 5. Lancet 2010; 375: 1609-23.

2. Ronsmans C, Graham WJ. Maternal mortality: who, when, where, and why. Lancet 2006;368:1189-200.

3. WHO Recommendations for the prevention of postpartum haemorrhage. Geneva: World Health Organisation, 2007.

4. Okamoto S, Hijikata-Okunomiya A, Wanaka K, Okada Y, Okamoto U. Enzyme controlling medicines: introduction. Semin Thromb Hemost 1997; 23: 493-501.

5. Henry DA, Carless PA, Moxey AJ, et al. Antifibrinolytic use for minimising perioperative allogeneic blood transfusion. Cochrane Database Syst Rev 2007; 4: CD001886.
6. The Lancet. CRASH-2: tranexamic acid and trauma patients [Online]. 2010 Jun 14 [cited 2010 Jun 20]; Available from: URL:http:/ / www. thelancet.com/ crash-2

7. CRASH-2 trial collaborators. Effects of tranexamic acid on death, vascular occlusive events, and blood transfusion in trauma patients with significant haemorrhage (CRASH-2): a randomised, placebocontrolled trial. Lancet. 2010 Jun 14;376(9734):23-32.

8. Ferrer P, Roberts I, Sydenham E, Blackhall K, Shakur $\mathrm{H}$. Anti-fi brinolytic agents in postpartum haemorrhage: a systematic review. BMC Pregnancy Childbirth 2009; 9: 29.

9. Shakur H, Elbourne D, Gülmezoglu M, Alfirevic Z, Ronsmans C, Allen E, et al. The WOMAN Trial (World Maternal Antifibrinolytic Trial): tranexamic acid for the treatment of postpartum haemorrhage: an international randomised, double blind placebo controlled trial. Trials. 2010 Apr 16;11:40. 